\title{
Unobtrusive, Through-Clothing ECG and Bioimpedance Monitoring in Sleep Apnea Patients
}

\author{
Ivan Castro $^{1}$, Aakash Patel ${ }^{1}$, Margot Deviaene ${ }^{2}$, Dorien Huysmans ${ }^{2}$, Pascal Borzée ${ }^{3}$, Bertien Buyse ${ }^{3}$, \\ Dries Testelmans 3 , Sabine Van Huffel $^{2}$, Carolina Varon ${ }^{2,4}$, Tom Torfs ${ }^{1}$ \\ ${ }^{1}$ imec, Leuven, Belgium \\ ${ }^{2}$ KU Leuven, Dept. Of Electrical Engineering (ESAT), Division Stadius, Leuven, Belgium \\ ${ }^{3}$ Leuven University Centre for Sleep and Wake Disorders, UZ Leuven, Leuven, Belgium \\ ${ }^{4}$ Circuits and Systems (CAS) group, Delft University of Technology, the Netherlands
}

\begin{abstract}
A real-life validation of a system for simultaneous acquisition of capacitively-coupled ECG (ccECG) and capacitively-coupled bioimpedance (ccBioz) is presented. The heart rate (HR) and respiration rate (RR) estimation performance was evaluated using polysomnography (PSG) signals as ground-truth, in recordings from 28 patients with suspected obstructive sleep apnea (OSA). A ccECG beat detection sensitivity of $98.4 \%$ and an $R-R$ interval mean absolute error (MAE) of $17.1 \mathrm{~ms}$ were achieved when applying quality-based algorithms. RR MAE values of 3.48 and 6.37 breaths per minute were also achieved when using two different RR extraction methods. High similarity between unobtrusive signals and PSG ground-truth was observed, with a correlation between ccECG and psgECG of $91.5 \%$ and a correlation between ccBioz and PSG thoracic belt (TB) of $89.5 \%$. Even in episodes containing OSA events, the characteristic respiration behavior of TB signals was also observed in the ccBioz signals. This shows the potential of ccECG and ccBioz for use in long-term monitoring without adding discomfort to the patient or user. Sleep-related applications as well as more generic cardiorespiratory monitoring in (patient) beds are obvious applications, but also other daily life monitoring can be done using a similar approach (e.g. in seats).
\end{abstract}

\section{Introduction}

Unobtrusive measurement of vital signs has been a topic of high interest in recent research [1]. The use of capacitively-coupled ECG (ccECG), for instance, has been proposed as a solution that allows long-term monitoring and can achieve results similar to medical grade ECG [2]. Similarly, capacitively-coupled bioimpedance (ccBioz) for impedance pneumography (IPG) can, when combined with ccECG, enable extended cardiorespiratory monitoring in daily life for preventive screening and patient follow-up.

One of the main challenges in the use of these technologies is the presence of motion artefacts (MA) [2]. To partially overcome this, researchers have proposed multiple solutions, with the use of signal quality indicators (SQIs) [3], [4] being more suitable for real-life scenarios.

Validation of ccECG in real-life scenarios in literature include its use for monitoring from a car seat [5], [6], from a bed [7], [8], among others. In the case of sleep monitoring, some of the evaluations include simultaneous polysomnography (PSG) measurements [7] from healthy subjects, but not from a population with sleep related disorders such as obstructive sleep apnea (OSA).

In the case of ccBioz, most results have been limited to experiments under controlled conditions [9] or in dummy models [10]. A real-life respiration monitoring evaluation from car drivers was presented by the authors in [6].

In [11], the authors presented a system with a ccECG array for SQI-based real-time electrode selection and simultaneous ccBioz monitoring, together with an initial sleep monitoring validation in healthy volunteers.

In this work, the ccECG \& ccBioz system from [11] is further validated in the clinic by monitoring 28 patients with suspected OSA, while simultaneously acquiring PSG signals. This, with two main goals: evaluating the heart rate (HR) and respiration rate (RR) estimation performance using PSG signals as ground-truth, as well as identifying the potential use of the ccECG and ccBioz unobtrusive signals in respiratory and cardiac applications.

\section{Methods}

\subsection{Data collection \& signal preprocessing}

The system for ccECG and ccBioz presented in [11] was 


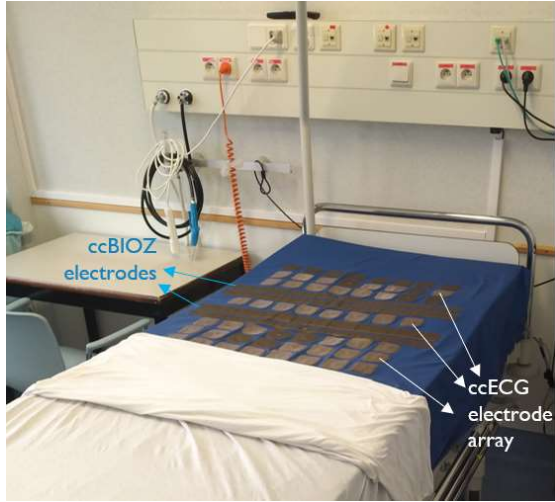

Figure 1. Mattress with ccECG and ccBioz sensors installed at the sleep laboratory. Cover partially removed for the picture (bed fully covered when collecting data).

installed in the sleep laboratory of the University Hospitals Leuven, Leuven, Belgium, as shown in Fig. 1. ccECG and ccBioz signals were acquired through a bed cover and through the patient's clothing simultaneously with standard PSG recordings, after signed informed consent. During the recordings, an algorithm for SQI-based electrode selection [3], [11] continuously selected the best set of electrodes to obtain 4 simultaneous ccECG traces.

The simultaneous PSG, ccECG and ccBIOZ signals were collected from 28 patients with suspected OSA during one night. From these, ccBioz signals were only available for the first 11 cases due to data loss in the remaining ones. A total of 295.2 hours of ccECG data and 116.1 hours of ccBioz data were acquired.

Signal pre-processing was applied as follows: ccECG and psgECG signals were filtered in the [0.67-40] Hz band, according to ambulatory ECG standards (IEC 60601-247). ccBioz and PSG respiratory signals from a thoracic belt (TB) were low pass filtered at $1 \mathrm{~Hz}$. To allow comparison between unobtrusive and PSG signals, the signals were aligned by means of the R-R interval series from ccECG and psgECG.

\subsection{Performance evaluation}

With the purpose of evaluating the performance of ccECG and ccBioz signals, the PSG signals (psgECG and psgTB) were used as ground-truth. All signals were divided into non-overlapping 60-second segments, for which apneic event annotations were available.

For each (ccECG \& psgECG) segment, ECG beat detection was performed using a Wavelet-based method and beat-to-beat intervals were extracted, as well as average heart rate (HR) per window. For each window, the respiration rate $(\mathrm{RR})$ values were obtained from $\mathrm{ccBioz}$ and TB signals by two different methods: 1) a frequencybased method detecting the maximum power spectral density (PSD) value in the [6-44] breaths per minute range, and 2) a time-domain method that detects the respiration peaks and obtains the averaged window RR from the breath-to-breath individual intervals.

The performance of the ccECG signals was evaluated for each patient by calculating, for each window, the beat detection sensitivity (i.e. match range of $\pm 75 \mathrm{~ms}$ ), positive predictive value (PPV), the R-R interval mean absolute error (MAE) and the tachogram correlation. The averaged metrics across windows were obtained per patient, as well as the MAE of the windowed HR values. These metrics were calculated when applying a SQI-based postprocessing approach that discards data subsegments with low quality in a 4-step approach [6], as well as when no SQI-based processing was applied. psgECG segments with low quality were not included in the analysis.

The ccBioz signals were evaluated by comparing the extracted RR by both the frequency-domain and timedomain methods described above and computing the MAE with respect to the equivalent extraction in the TB signals. No SQI-based processing or manual signal discarding was applied in this case. In addition to the RR evaluation, the correlation between the unobtrusive and ground-truth signals in segments with good signal quality was obtained, and a visual inspection of some of the segments labelled as containing apneic episodes was done, to identify the potential of using these signals for automatic identification of OSA epochs.

\section{Results and discussion}

The overall results when comparing ccECG to psgECG signals are shown in Table 1, both when applying and when not applying the SQI-based signal processing. The results of the ccBioz comparison against the TB for extraction of RR are shown in Table 2.

$\mathrm{R}-\mathrm{R}$ interval root mean squared error (RMSE) from

Table 1. Overall averaged performance metrics of ccECG compared against psgECG. Evaluation done in 60-second non-overlapping windows.

\begin{tabular}{|c|c|c|c|c|c|c|}
\hline Data & $\begin{array}{c}\text { Sens. } \\
\text { (\%) }\end{array}$ & $\begin{array}{c}\text { PPV } \\
\text { (\%) }\end{array}$ & $\begin{array}{c}\text { R-R } \\
\text { MAE } \\
\text { (ms) }\end{array}$ & $\begin{array}{c}\text { TC } \\
\text { (\%) }\end{array}$ & $\begin{array}{c}\text { Window } \\
\text { HR MAE } \\
\text { (bpm) }\end{array}$ & $\begin{array}{c}\text { Used } \\
\text { data } \\
\text { (\%) }\end{array}$ \\
\hline $\begin{array}{c}\text { ccECG } \\
\text { (No } \\
\text { SQI) }\end{array}$ & 64.9 & 63.8 & $319.2^{1}$ & 87.1 & 19.8 & 100 \\
\hline $\begin{array}{c}\text { ccECG } \\
\text { (with } \\
\text { SQI) }\end{array}$ & 98.4 & 98.0 & $17.1^{2}$ & 99.6 & 1.5 & $\begin{array}{c}{[19.3} \\
- \\
73.2]^{3}\end{array}$ \\
\hline
\end{tabular}

TC: Tachogram Correlation; Sens: Sensitivity;

PPV: Positive Predictive Value; MAE: Mean Absolute Error

${ }^{1}$ RMSE: 19.8ms; ${ }^{2}$ RMSE: $1.5 \mathrm{~ms}$

${ }^{3}$ Range of per-night data used. Median of 37.1; Mean 38.5 
Table 2. Overall averaged performance metrics of respiration rate from ccBioz compared against respiration rate from TB signals

\begin{tabular}{|c|c|c|c|}
\hline Data & $\begin{array}{c}\text { Window } \\
\text { RR MAE } \\
\text {-Freq. } \\
\text { based- } \\
\text { (bpm) }\end{array}$ & $\begin{array}{c}\text { Window } \\
\text { RR MAE } \\
\text {-Time } \\
\text { based- } \\
\text { (bpm) }\end{array}$ & $\begin{array}{c}\text { Used } \\
\text { data } \\
\text { (\%) }\end{array}$ \\
\hline ccBIOZ & 3.48 & 6.37 & 100 \\
\hline
\end{tabular}

ccECG after applying SQI-based processing is $1.5 \mathrm{~ms}$ $(\mathrm{MAE}=17.1 \mathrm{~ms})$, which is only slightly higher than the RMSE of $1.36 \mathrm{~ms}$ reported in literature for healthy subjects [7]. The obtained R peak sensitivity of $98.4 \%$ is similar to the $98.0 \%$ presented in the same study. Nevertheless, there is a lower amount of used data due to the MAs caused by motion or low electrode coupling.

It is clear from the ccECG results in Table 1 that the SQI-based signal processing is crucial to obtain R-R intervals and $\mathrm{HR}$ values in agreement with the psgECG, at the expense of using a subset of the recorded ccECG data. This, because the ccECG signals present parts of lower quality that can be caused by movement, position change and PSG bands ( 3 in total) overlapping with the electrodes.

The per-night subset of high-quality ccECG data for these experiments after SQI-based signal processing was in the range of [19.3-73.2]\%. This shows important differences in the amount of high-quality data between patients/nights, which may be related to the amount of motion during a specific night. This can be caused in some cases by the awakening after an apnea period. To verify the latter, the percentage of segments labelled with an OSA event per night were plotted against the percentage of highquality data as shown in Fig. 2 .

From this figure it can be seen that although a subset of patients show an inversely proportional relation between

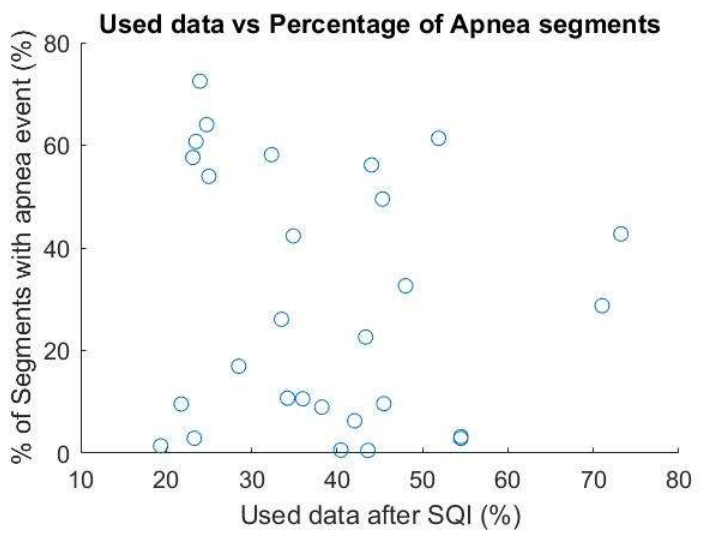

Figure 2. Comparison across the recorded nights between percentage of high-quality ccECG data used after applying SQI algorithms and the percentage of segments labelled with an OSA event per night. segments containing apneic events and used data, there are cases in which a relatively high percentage of apneic events did not result in a low amount of used data, and others in which a low amount of used data correspond to nights with low percentage of OSA segments. This points out why the apnea-related movements are only partially causing low-quality signals. The presence of PSG belts and night-specific increased movement (possibly due to a different sleeping scenario) is therefore another important part of the cause.

Consequently, the amount of high-quality ccECG data in a home environment is expected to be higher than what was obtained in this study. This was initially verified with 10 overnight measurements of home monitoring from a healthy volunteer, which resulted in an average coverage of $\sim 60 \%$ and a range of $[44.6-80.1] \%$.

These results suggest that a combination of the increased motion (by both the altered sleep setting and some of the OSA-related awakenings) and the presence of the OSA belts can cause lower coverage of this technology than when used in a home setting without a PSG system.

A similar effect of movement and PSG bands is present in the ccBioz signals. In this case, RR MAE values obtained were $3.48 \mathrm{bpm}$ and $6.37 \mathrm{bpm}$. These are
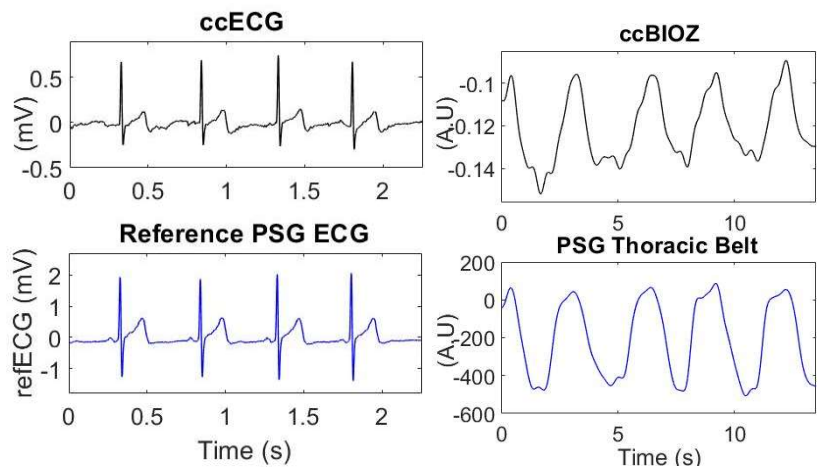

(a)

(b)

Figure 3. Comparison between morphology of ccECG and psgECG signals (a) $-91.5 \%$ correlation- and between the ccBioz and psgTB signals (b) $-89.5 \%$ correlation-. Both signals present high similarity in segments with good signal quality.

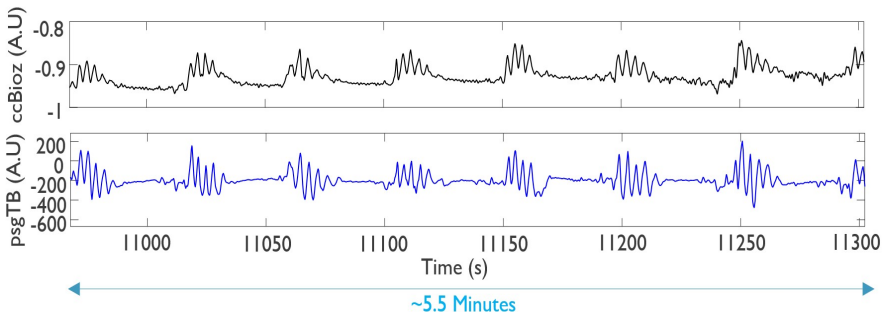

Figure 4. Comparison between the respiratory signals based on ccBioz (Top) and TB from PSG (bottom) in a $~ 5.5$ minute segment with multiple apneic episodes. 
promising results that show the potential of the signals for RR extraction, but are still relatively high, considering a 'normal' physiological range of [6-44] bpm.

In the future, ccBioz SQI algorithms as well as an array of ccBioz electrodes, together with modifications in the Bioz circuitry to further optimize it for the non-contact case, have the potential to improve the respiration results. This, not only with the purpose of RR extraction, but also with the aim of identifying specific respiratory conditions or events such as OSA epochs.

When making a comparison of the morphology of the signals, it was identified that segments with high quality data showed high similarity with the PSG ground-truth signals, as can be seen in Fig. 3. A correlation of $91.5 \%$ was obtained between the ccECG segment and the psgECG reference shown in the figure. Similarly, a comparison between ccBioz-based and psgTB-based respiratory activity signals shown, resulted in a correlation of $89.5 \%$. This agrees with previous proof-of-concept experiments where the ccECG waveform achieved up to $98 \%$ correlation when compared to medical-grade contact ECG and ccBioz signals followed respiration patterns.

In the case of segments with OSA events, it was seen that the characteristic respiratory behaviour of TB signals is also reflected in ccBioz signals. This is seen in Fig. 4, where the periodic respirations followed by apneic periods are clearly visible in the ccBioz measurement.

\section{Conclusions}

This work presented a real-life validation of a system for the unobtrusive acquisition of ccECG and ccBiozbased respiratory activity on OSA patients when compared to PSG signals as ground-truth.

High performance of ccECG signals was achieved in terms of beat detection sensitivity, R-R interval error and signal correlation to ground-truth. This was achieved by applying SQI-based algorithms, found to be necessary to automatically identify high-quality data and significantly lower errors in extracted metrics.

ccBioz signals also achieved a high correlation with ground-truth reference in segments of good quality and promising results in terms of RR MAE. Additional work related to the Bioz acquisition system and SQI algorithms for ccBioz are necessary and part of future work.

Further exploration of ccECG and ccBioz signals for application-specific purposes such as the automatic detection of OSA epochs is necessary, including the evaluation of related features. Furthermore, the high similarity between the unobtrusive ccECG and ccBioz to ground-truth PSG signals demonstrates the feasibility of the use of these technologies for long-term monitoring in multiple scenarios, not only for sleep monitoring, but also in different real-life scenarios such as driver monitoring, office monitoring, among others.

\section{Acknowledgments}

Agentschap Innoveren en Ondernemen (VLAIO) 150466: OSA+ and O\&O HBC 20160184 eWatch. Imec funds. This research received funding from the Flemish Government (AI Research Program). SVH, MD, CV, DH are affiliated to Leuven.AI - KU Leuven institute for AI, B-3000, Leuven, Belgium.

\section{References}

[1] C. Brüser et al., "Ambient and unobtrusive cardiorespiratory monitoring techniques," IEEE Rev. Biomed. Eng., vol. 8, pp. 30-43, 2015.

[2] Y. Sun and X. B. Yu, "Capacitive biopotential measurement for electrophysiological signal acquisition: A Review," IEEE Sens. J., vol. 16, no. 9, pp. 2832-2853, 2016.

[3] I. Castro et al., "Data quality assessment of capacitivelycoupled ECG signals," in Computers in Cardiology, 2019 , p. 4.

[4] K. Kido et al., "A novel CNN-based framework for classification of signal quality and sleep position from a capacitive ECG measurement," Sensors, vol. 19, no. 7, p. 1731, 2019.

[5] T. Wartzek, et al., "ECG on the road: robust and unobtrusive estimation of heart rate," IEEE Trans. Biomed. Eng., vol. 58, no. 11, pp. 3112-3120, Nov. 2011.

[6] I. D. Castro et al., "Physiological driver monitoring using capacitively coupled and radar sensors," Appl. Sci., vol. 9, no. 19, 2019.

[7] H. J. Lee et al., "Heart rate variability monitoring during sleep based on capacitively coupled textile electrodes on a bed," Sensors, vol. 15, no. 5, pp. 11295-11311, May 2015.

[8] M. Takano and A. Ueno, "Noncontact in-bed measurements of physiological and behavioral signals using an integrated fabric-sheet sensing scheme," IEEE J. Biomed. Heal. Informatics, vol. 23, no. 2, pp. 618 630, 2019.

[9] R. Macías et al., "Ventilation and heart rate monitoring in drivers using a contactless electrical bioimpedance system," J. Phys. Conf. Ser., vol. 434, no. 1, p. 012047 , Apr. 2013.

[10] D. G. Abad, "Development of a capacitive bioimpedance measurement system," RWTH Aachen University, 2009.

[11] I. D. Castro et al., "Capacitive multi-electrode array with real-time electrode selection for unobtrusive ECG \& BIOZ monitoring," in Eng. in Med. \& Biol. Conf., $E M B C, 2019$

Address for correspondence:

Ivan D. Castro

imec: Kapeldreef 75, 3001 Leuven, Belgium

ivand.castro@imec.be 